\title{
Mean Performance, Character Association and Path Analysis Studies for Quantitative Characters in Okra (Abelmoschus esculentus (L.) Moench) Genotypes
}

\author{
R. Kiran Kumar* and Ramangouda V. Patil \\ University of Agricultural Scinces, Dharwad, Karnataka, India \\ *Corresponding author
}

\begin{abstract}
A B S T R A C T
Keywords

Abelmoschus esculentus (L.)

Moench,

Correlation coefficients, Path analysis and Genotypes

Article Info

Accepted:

12 October 2020

Available Online:

10 November 2020

The present investigation was carried out to study the mean performance, character association and path coefficient analysis in okra (Abelmoschus esculentus (L.) Moench) during the kharif season of 2019-20. The experiment was laid out in Randomized Block Design with two replications at Olericulture Unit, Department of Horticulture, Main Agricultural Research Station, UAS, Dharwad. In the present study, the genotypes 360-R, IC-014096, 29-2-2, IC-00795, IC-755647 and 10-191 recorded high mean performance for fruit yield per plant. Significant and positive correlation was observed for number of fruits per plant, average fruit weight, fruit length, plant height 90 DAS, plant height 30 DAS, plant height 60 DAS and fruit diameter with fruit yield per plant. This indicates the possibility in increasing the yield by selecting for these characters. Path coefficient analysis indicated that, the number of fruits per plant had highest positive direct effect on fruit yield per plant followed by average fruit weight, days to fifty per cent flowering, internodal length, number of branches per plant, fruit length, fruit diameter, days to first flowering and plant height 60 DAS. This further indicates the positive correlation between these characters.

\section{Introduction}

Bhendi (Abelmoschus esculentus (L.) Moench) is one of the important tropical vegetable crops commonly known as okra and lady finger in India. It is the most ancient and traditional crop grown in tropical and subtropical low land regions of Asia, Africa, America and warmer parts of Mediterranean regions (Chauvahan, 1972).

According to the taxonomic classification of Zeven and Zhukovsky (1975), the cultivated

species A. esculentus is believed to have originated in the Hindustani centre, i.e., India. The first step in okra improvement should involve evaluation of the germplasm to assess the mean performance for yield and yield related traits. If there are any lines which would be performing better than commercial check, such lines could be pushed for commercial exploitation. A study conducted by Thirupathi et al., (2012), indicated that ten entries performed superior as compared to commercial check Arka Anamika. Kumar et al., (2019), evaluated sixty eight genotypes
\end{abstract}


and reported that two entries such as B-02 and $\mathrm{KS}-442$ were superior as compared to other entries. Nesru et al., (2020), studied thirtythree okra genotypes for twenty seven quantitative characters and reported higher fruit yield per hectare in the genotype 29618 as compared to other genotypes.

Correlation studies indicate the degree of interrelationship of plant characters for improvement of yield as well as important quality parameters in any breeding programme. Hence, understanding of the interrelationship between yield and yield influencing characters is of vital importance because this would facilitate effective selection for simultaneous improvement in one or more yield characters. Path coefficient analysis facilitates the partitioning of correlation coefficient into direct and indirect effects of various traits on fruit yield per plant. It provides an effective means of finding out direct and indirect causes of association and provides a critical examination of the specific forces acting to produce a given correlation and measures the relative importance of each factor. Mishra et al., (2016), reported that fruit yield per plant had highly significant and positive association with number of nodes per plant, number of fruits per plant, days to first flowering, internodal length, number of branches per plant and plant height. Saryam et al., (2017), evaluated okra genotypes and reported that yield per plant had positive association with fruit diameter, fruit length, number of fruits per plant, fruit weight, number of seeds per fruit, 100 seed weight and fruit diameter. Kumar et al., (2019), studied sixty eight genotypes of okra for different yield contributing characters and reported that total fruit yield per plant had positive direct effect with fruit girth, number of fruits per plant, fruit weight and internodal distance. Hence the present study was conducted to realize the importance of developing high yielding okra genotypes.

\section{Materials and Methods}

The investigation was carried out at Olericulture Unit, Department of Horticulture. MARS, UAS, Dharwad during kharif 201920. The experimental material which is used in the present investigation comprised of fifty genotypes of okra. They were evaluated using randomized block design (RBD). Twenty six genotypes were collected from NBPGR Hyderabad, twenty two from UAS, Dharwad and two from IIHR Bangalore. The row to row spacing was $60 \mathrm{~cm}$ and plant to plant spacing was $30 \mathrm{~cm}$. The recommended package of practices by UHS, Bagalkot was followed. Five plants were selected randomly from each replication and data were recorded for the characters viz., plant height 30 DAS $(\mathrm{cm})$, plant height 60 DAS $(\mathrm{cm})$, plant height 90 DAS $(\mathrm{cm})$, days to first flowering, days to fifty per cent flowering, number of branches per plant, internodal length $(\mathrm{cm})$, first fruiting node, days to first harvest, number of fruits per plant, number of seeds per fruit, fruit length $(\mathrm{cm})$, average fruit weight $(\mathrm{g})$, fruit diameter $(\mathrm{cm})$, yield per plant $(\mathrm{g})$ and yield per hectare (t). Genotypic and phenotypic correlation coefficients were calculated using the method given by Johnson et al., (1955). Path coefficient analysis was carried out by the procedure originally proposed by Wright (1921).

\section{Results and Discussion}

In the present study it is evident from the Table 1, that there was significant variation in the mean performance of the fifty genotypes for all the sixteen traits studied. The most important trait in any crop improvement programme is the fruit yield. Significantly higher yield was recorded in the entry 360-R (443.42 g) and there were only two entries namely IC-014096 and Arka Anamika which were at par with this (Table 1-3). 
Table.1 Mean performance of the fifty genotypes for growth and yield characters in okra

\begin{tabular}{|c|c|c|c|c|c|c|c|c|c|c|c|c|c|c|c|c|c|}
\hline $\begin{array}{l}\dot{Z} \\
\dot{\tilde{B}}\end{array}$ & 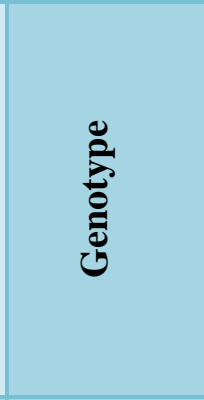 & 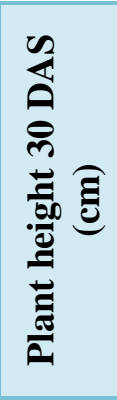 & 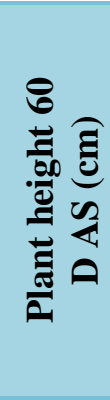 & 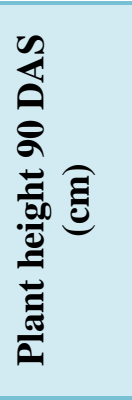 & 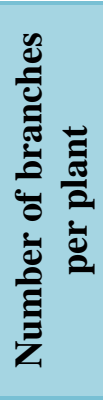 & 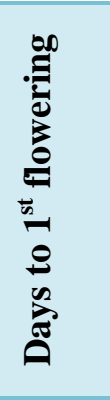 & 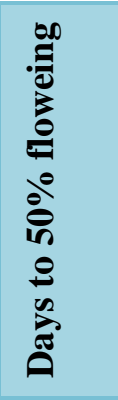 & 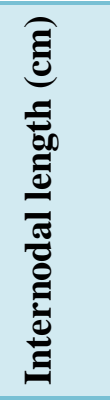 & 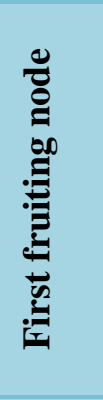 & 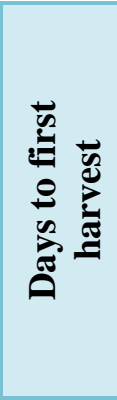 & 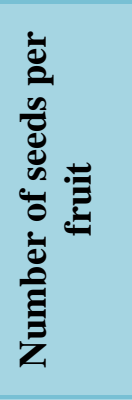 & 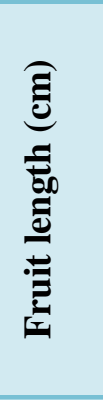 & 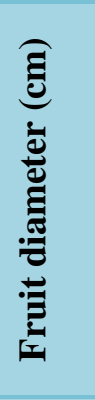 & 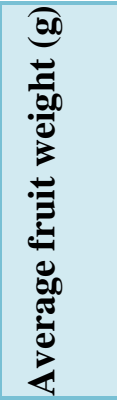 & 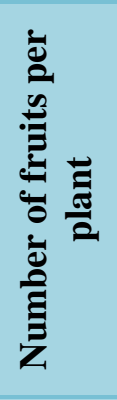 & 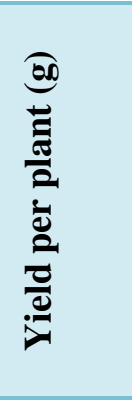 & 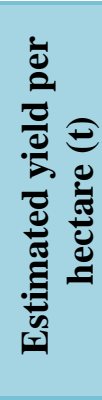 \\
\hline 1 & IC-014018 & 46.20 & 80.70 & 116.90 & 1.00 & 49.52 & 52.59 & 8.80 & 4.16 & 54.50 & 75.25 & 12.84 & 5.08 & 16.30 & 15.75 & 235.25 & 8.04 \\
\hline 2 & IC-009856 & 49.10 & 87.25 & 166.10 & 2.30 & 52.30 & 51.43 & 5.50 & 5.50 & 57.16 & 48.50 & 12.79 & 5.35 & 14.54 & 17.20 & 215.97 & 7.17 \\
\hline 3 & IC-015540 & 4.40 & 76.75 & 156.65 & 1.00 & 47.52 & 49.88 & 6.03 & 3.83 & 54.16 & 122.75 & 12.41 & 7.71 & 15.24 & 16.05 & 212.61 & 7.51 \\
\hline 4 & IC-755647 & 47.30 & 87.75 & 166.95 & 2.45 & 48.06 & 51.46 & 7.81 & 5.83 & 54.83 & 83.00 & 14.90 & 5.51 & 18.73 & 18.95 & 354.81 & 11.8 \\
\hline 5 & IC-42490 & 50.05 & 75.88 & 148.45 & 1.00 & 39.89 & 40.94 & 16.35 & 4.67 & 45.99 & 56.75 & 16.41 & 5.79 & 17.15 & 15.80 & 270.99 & 9.02 \\
\hline 6 & IC-433641 & 38.65 & 64.10 & 127.50 & 1.45 & 53.15 & 54.57 & 11.50 & 4.83 & 57.83 & 37.75 & 13.40 & 5.36 & 12.91 & 14.90 & 218.81 & 7.06 \\
\hline 7 & IC-011533 & 41.20 & 74.20 & 129.20 & 1.00 & 48.57 & 53.42 & 7.36 & 4.83 & 55.49 & 47.25 & 14.40 & 5.11 & 12.46 & 20.60 & 256.47 & 8.53 \\
\hline 8 & IC-111515 & 41.80 & 78.75 & 145.10 & 2.80 & 52.66 & 55.78 & 8.83 & 4.83 & 58.66 & 70.75 & 13.10 & 5.37 & 12.11 & 16.95 & 219.31 & 6.86 \\
\hline 9 & IC-016566 & 5.65 & 92.50 & 148.12 & 3.45 & 51.92 & 54.18 & 9.05 & 5.33 & 58.16 & 56.25 & 13.31 & 6.49 & 16.80 & 15.40 & 258.62 & 8.60 \\
\hline 10 & IC-00795 & 52.75 & 99.95 & & 4.80 & 40.34 & 43.41 & 10.38 & 6.50 & 46.49 & 57.52 & 14.52 & 5.97 & 18.33 & 19.50 & 357.40 & 11.91 \\
\hline 11 & IC-008769 & 48.20 & 89.65 & 148.80 & 1.80 & 52.09 & 53.82 & 7.11 & 5.50 & 57.33 & 56.75 & 13.26 & 6.22 & 17.20 & 18.30 & 314.84 & 10.48 \\
\hline 12 & IC-04328 & 55.60 & 83.60 & 141.35 & 1.45 & 42.97 & 45.43 & 9.30 & 3.83 & 48.49 & 74.25 & 15.42 & 5.70 & 16.48 & 18.90 & 311.52 & 10.37 \\
\hline 13 & IC-001543 & 42.75 & 81.95 & 142.25 & 1.00 & 48.71 & 51.82 & 8.26 & 3.83 & 54.33 & 91.75 & 14.96 & 7.11 & 21.72 & 13.90 & & 10.04 \\
\hline 14 & IC-014096 & 44.55 & 86.45 & 141.05 & 1.65 & 43.32 & 46.83 & 7.62 & 4.67 & 49.83 & 43.75 & 18.09 & 5.40 & 18.89 & 21.50 & & 14.15 \\
\hline 15 & IC-014600 & 52.35 & 88.65 & 139.95 & 1.00 & 46.94 & 49.72 & 9.26 & 4.50 & 52.83 & 50.75 & 15.50 & 5.04 & 14.89 & 20.70 & 308.18 & 10.26 \\
\hline 16 & IC-013995 & 55.22 & 82.75 & 132.90 & 1.30 & 39.32 & 41.59 & 10.99 & 3.33 & 45.83 & 52.25 & 15.10 & 5.09 & 13.42 & 12.90 & 160.17 & 5.33 \\
\hline 17 & IC-42404 & 45.75 & 80.20 & 128.10 & 1.00 & 49.91 & 52.27 & 11.47 & 3.50 & 56.16 & 53.25 & 14.10 & 6.16 & 15.55 & 14.65 & 242.80 & 8.24 \\
\hline 18 & IC-0113356 & 49.10 & 85.10 & 166.05 & 1.00 & 48.42 & 50.21 & 7.15 & 4.83 & 53.83 & 56.25 & 17.17 & 5.20 & 11.96 & 15.00 & 204.86 & 7.08 \\
\hline 19 & IC-90219 & 46.25 & 78.20 & 150.85 & 1.45 & 49.77 & 54.59 & 8.97 & 5.50 & 57.00 & 62.25 & 15.36 & 6.25 & 18.14 & 16.60 & 300.99 & 10.02 \\
\hline 20 & IC-04328 & 38.15 & 77.95 & 132.00 & 1.45 & 49.10 & 53.84 & 7.16 & 4.50 & 56.33 & 61.00 & 15.92 & 5.68 & 16.58 & 15.90 & 263.67 & 8.77 \\
\hline 21 & IC-29119 & 42.30 & 78.95 & 119.60 & 1.00 & 44.44 & 48.61 & 11.69 & 3.50 & 51.33 & 89.00 & 11.09 & 5.57 & 18.66 & 14.40 & 268.76 & 8.93 \\
\hline 22 & IC-10265 & 47.25 & 85.50 & 152.20 & 2.30 & 52.76 & 54.05 & 8.94 & 5.50 & 57.66 & 57.00 & 12.87 & 6.41 & 14.96 & 16.60 & 248.51 & 8.26 \\
\hline 23 & RHBG-1 & 42.33 & 87.40 & 118.55 & 1.45 & 45.98 & 46.57 & 9.95 & 4.50 & 50.83 & 52.25 & 13.75 & 5.70 & 18.60 & 9.20 & 171.06 & 5.68 \\
\hline
\end{tabular}




\begin{tabular}{|c|c|c|c|c|c|c|c|c|c|c|c|c|c|c|c|c|c|}
\hline 24 & RHBG-7 & 52.70 & 85.60 & 137.55 & 1.45 & 45.39 & 48.61 & 12.43 & 3.83 & 52.16 & 43.50 & 13.62 & 5.77 & 15.25 & 13.75 & 231.67 & 7.64 \\
\hline 25 & RHBG-43 & 43.50 & 86.75 & 101.25 & 4.80 & 46.77 & 46.75 & 10.90 & 3.50 & 52.16 & 78.25 & 12.22 & 6.01 & 14.00 & 15.10 & 224.88 & 7.46 \\
\hline 26 & RHBG-175-41 & 45.52 & 86.82 & 125.75 & 3.45 & 50.00 & 53.00 & 12.01 & 3.50 & 56.00 & 108.75 & 13.34 & 5.66 & 15.05 & 17.00 & 255.84 & 8.51 \\
\hline 27 & VRD-6 & 45.10 & 83.60 & 133.75 & 1.45 & 47.83 & 50.50 & 11.25 & 3.50 & 53.83 & 62.25 & 13.24 & 5.83 & 14.00 & 14.20 & 198.82 & 6.60 \\
\hline 28 & $20-298$ & 47.45 & 82.70 & 152.60 & 1.30 & 50.00 & 53.00 & 8.88 & 5.50 & 56.00 & 55.75 & 15.22 & 5.66 & 17.13 & 14.70 & 251.74 & 8.37 \\
\hline 29 & $29-2-2$ & 43.50 & 84.55 & 156.45 & 2.30 & 52.16 & 55.00 & 7.48 & 6.16 & 58.16 & 81.75 & 15.02 & 6.17 & 17.98 & 21.80 & 391.76 & 13.04 \\
\hline 30 & $36-377$ & 43.05 & 82.88 & 138.22 & 1.80 & 46.50 & 49.50 & 10.61 & 4.50 & 52.49 & 76.25 & 11.94 & 5.68 & 13.18 & 16.20 & 212.03 & 7.02 \\
\hline 31 & $40-1$ & 53.35 & 99.45 & 171.65 & 1.00 & 45.83 & 48.50 & 7.41 & 6.50 & 51.83 & 62.75 & 12.95 & 6.45 & 18.33 & 16.30 & 298.94 & 9.95 \\
\hline 32 & $42-322$ & 48.15 & 88.80 & 163.15 & 1.45 & 49.16 & 52.50 & 10.83 & 4.83 & 55.16 & 82.75 & 13.20 & 6.37 & 16.69 & 18.30 & 305.45 & 10.17 \\
\hline 33 & $59-112$ & 43.69 & 80.50 & 111.80 & 1.00 & 46.66 & 49.50 & 11.13 & 3.67 & 52.16 & 72.25 & 13.47 & 5.83 & 14.05 & 16.10 & 236.23 & 7.97 \\
\hline 34 & $10-191$ & 46.55 & 86.20 & 154.70 & 1.00 & 45.83 & 48.50 & 9.22 & 3.83 & 51.33 & 67.25 & 14.49 & 5.68 & 18.15 & 19.30 & 350.37 & 11.66 \\
\hline 35 & NO:69 & 50.85 & 88.20 & 174.05 & 3.50 & 53.83 & 56.50 & 7.24 & 5.83 & 59.83 & 66.75 & 14.81 & 5.03 & 13.14 & 17.50 & 230.01 & 7.64 \\
\hline 36 & NO:32 & 43.95 & 88.35 & 171.90 & 1.80 & 52.33 & 55.50 & 7.01 & 4.83 & 58.33 & 64.00 & 14.01 & 5.90 & 17.18 & 13.60 & 233.57 & 7.77 \\
\hline 37 & 279-R & 50.10 & 92.60 & 170.60 & 3.15 & 54.16 & 57.00 & 6.20 & 5.50 & 60.16 & 59.50 & 11.94 & 5.42 & 12.33 & 18.20 & 239.90 & 8.35 \\
\hline 38 & $360-R$ & 65.30 & 89.75 & 173.10 & 1.00 & 49.67 & 51.50 & 7.16 & 3.83 & 55.66 & 60.25 & 15.91 & 6.39 & 21.92 & 22.50 & 443.42 & 14.75 \\
\hline 39 & Anamika $\times 43$ & 53.05 & 88.90 & 154.30 & 1.45 & 53.00 & 56.00 & 7.86 & 4.83 & 59.00 & 63.25 & 16.07 & 5.17 & 17.48 & 17.20 & 300.56 & 10.00 \\
\hline 40 & Abhay $\times 43$ & 54.75 & 99.85 & 177.90 & 1.45 & 49.33 & 51.50 & 9.72 & 3.83 & 55.33 & 76.75 & 14.67 & 5.68 & 15.14 & 19.20 & 290.78 & 9.68 \\
\hline 41 & $43 \times$ Abhay & 54.75 & 97.25 & 170.80 & 1.45 & 50.33 & 53.50 & 7.99 & 4.83 & 56.33 & 62.25 & 16.29 & 5.46 & 15.54 & 19.20 & 298.16 & 9.93 \\
\hline 42 & B-2, $210(44 s)$ & 45.85 & 95.75 & 155.50 & 1.00 & 49.17 & 51.50 & 8.95 & 5.33 & 55.16 & 53.25 & 13.32 & 6.28 & 18.20 & 15.10 & 279.81 & 9.13 \\
\hline 43 & B-2, 14-2 (47s) & 52.55 & 98.10 & 165.25 & 1.00 & 45.50 & 48.50 & 10.64 & 6.67 & 51.50 & 57.25 & 13.61 & 6.14 & 18.94 & 17.60 & 333.35 & 11.08 \\
\hline 44 & B- $1,60,15$ & 47.10 & 94.27 & 168.25 & 1.00 & 46.50 & 49.50 & 11.71 & 3.83 & 52.49 & 75.75 & 13.42 & 6.16 & 17.15 & 14.20 & 243.63 & 8.11 \\
\hline 45 & $\mathrm{~B}-3,39 \mathrm{R}$ & 52.40 & 101.9 & 177.75 & 1.80 & 50.00 & 52.50 & 7.57 & 4.83 & 55.99 & 56.25 & 14.35 & 6.02 & 16.20 & 17.30 & 280.38 & 9.33 \\
\hline 46 & B-3, 149,22 & 53.95 & 98.90 & 168.00 & 2.30 & 50.50 & 53.00 & 7.83 & 4.83 & 56.49 & 56.75 & 13.79 & 6.57 & 18.38 & 18.30 & 336.38 & 11.2 \\
\hline 47 & $4 \times 5$ & 47.70 & 89.40 & 167.45 & 1.45 & 51.33 & 53.50 & 8.39 & 4.83 & 57.33 & 51.25 & 14.45 & 5.68 & 15.15 & 19.10 & 289.35 & 9.64 \\
\hline 48 & Arka Abhay & 52.10 & 95.00 & 155.15 & 1.30 & 46.83 & 49.50 & 11.66 & 3.83 & 52.83 & 66.75 & 15.00 & 5.69 & 16.79 & 19.20 & 322.39 & 10.73 \\
\hline 49 & Arka Anamika & 46.30 & 82.30 & 149.95 & 1.30 & 49.00 & 51.50 & 9.61 & 3.83 & 55.49 & 61.25 & 16.97 & 6.39 & 20.82 & 19.30 & 423.06 & 14.08 \\
\hline 50 & GS-303 & 42.00 & 73.70 & 104.93 & 1.00 & 46.17 & 48.50 & 8.17 & 3.50 & 51.60 & 55.75 & 15.65 & 6.29 & 12.95 & 13.90 & 180.02 & 5.97 \\
\hline \multicolumn{2}{|l|}{ Mean } & 47.83 & 86.52 & 149.03 & 1.72 & 48.43 & 51.03 & 9.18 & 4.64 & 54.41 & 46.69 & 14.27 & 5.85 & 16.25 & 16.87 & 276.10 & 9.08 \\
\hline \multicolumn{2}{|c|}{ C.V. } & 6.61 & 6.17 & 6.41 & 12.34 & 2.00 & 1.36 & 5.17 & 5.16 & 0.93 & 8.24 & 6.55 & 6.33 & 8.14 & 5.42 & 9.02 & 9.16 \\
\hline \multicolumn{2}{|c|}{ S.Em \pm} & 2.23 & 3.77 & 6.75 & 0.15 & 0.68 & 0.49 & 0.33 & 0.17 & 0.36 & 2.39 & 0.35 & 0.13 & 0.59 & 0.28 & 9.80 & 0.25 \\
\hline \multicolumn{2}{|c|}{ C.D. 5\% } & 6.35 & 10.73 & 19.20 & 0.42 & 1.95 & 1.39 & 0.95 & 0.48 & 1.02 & 6.81 & 1.02 & 0.39 & 1.68 & 0.82 & 27.87 & 1.19 \\
\hline \multicolumn{2}{|c|}{ C.D. $1 \%$} & 8.48 & 14.32 & 25.61 & 0.56 & 2.60 & 1.86 & 1.27 & 0.64 & 1.36 & 9.09 & 1.36 & 0.52 & 2.24 & 1.09 & 37.17 & 1.60 \\
\hline \multicolumn{2}{|c|}{ Range Lowest } & 38.15 & 64.10 & 101.25 & 1.00 & 39.32 & 40.94 & 5.50 & 3.33 & 45.83 & 37.75 & 11.09 & 5.03 & 11.96 & 9.20 & 160.17 & 5.33 \\
\hline \multicolumn{2}{|c|}{ Range Highest } & 65.30 & 101.9 & 181.45 & 4.80 & 54.16 & 57.00 & 16.35 & 6.67 & 60.16 & 122.75 & 18.09 & 7.71 & 21.92 & 22.50 & 443.42 & 14.75 \\
\hline
\end{tabular}


Table.2 Genotypic and phenotypic correlation coefficients among growth and yield parameters in okra

\begin{tabular}{|c|c|c|c|c|c|c|c|c|c|c|c|c|c|c|c|c|c|}
\hline & & $\mathbf{X 1}$ & $\mathbf{X} 2$ & $\mathbf{X} 3$ & $\mathrm{X} 4$ & X5 & X6 & $\mathrm{X} 7$ & X8 & X9 & X10 & X11 & X12 & X13 & X14 & X15 & X16 \\
\hline \multirow[t]{2}{*}{ X1 } & $\mathrm{rp}$ & 1.000 & $0.459 * *$ & $0.454 * *$ & -0.137 & -0.190 & 0.052 & -0.150 & -0.031 & $0.325^{* *}$ & $0.229 * *$ & 0.172 & -0.078 & -0.022 & -0.120 & $0.342 *$ & $0.339 * *$ \\
\hline & $\mathrm{rg}$ & 1.000 & 0.839 & 0.721 & -0.184 & -0.213 & 0.128 & -0.181 & -0.068 & 0.395 & 0.286 & 0.225 & -0.064 & -0.027 & -0.175 & 0.425 & 0.419 \\
\hline \multirow[t]{2}{*}{$\mathbf{X} 2$} & $\mathrm{rp}$ & & 1.000 & $0.623 * *$ & $0.001 * *$ & $0.015^{* *}$ & $0.305^{*}$ & 0.016 & -0.155 & $0.234 * *$ & -0.001 & $0.265^{*}$ & 0.095 & $0.205^{*}$ & -0.045 & $0.312 *$ & $0.309 * *$ \\
\hline & $\mathrm{rg}$ & & 1.000 & 0.708 & 0.046 & -0.027 & 0.413 & 0.012 & -0.199 & 0.306 & -0.078 & 0.316 & 0.170 & 0.312 & -0.050 & 0.390 & 0.386 \\
\hline \multirow[t]{2}{*}{$\mathbf{X 3}$} & $\mathrm{rp}$ & & & 1.000 & $0.201 *$ & $0.230 *$ & $0.519 * *$ & $0.239^{*}$ & $-0.343 * *$ & $0.355^{* *}$ & 0.154 & $0.233^{*}$ & -0.011 & 0.077 & -0.044 & $0.393^{*}$ & $0.356 * *$ \\
\hline & $\mathrm{rg}$ & & & 1.000 & 0.265 & 0.231 & 0.601 & 0.265 & -0.403 & 0.451 & 0.183 & 0.243 & -0.026 & 0.118 & -0.048 & 0.447 & 0.442 \\
\hline \multirow[t]{2}{*}{$\mathrm{X} 4$} & $\mathrm{rp}$ & & & & 1.000 & $0.934 * *$ & $0.264 *$ & $0.970 * *$ & $-0.500 * *$ & 0.101 & -0.170 & -0.170 & -0.056 & 0.175 & -0.044 & $0.393^{*}$ & -0.045 \\
\hline & $\mathrm{rg}$ & & & & 1.000 & 0.981 & 0.274 & 1.003 & -0.547 & 0.119 & -0.209 & -0.185 & -0.071 & 0.181 & -0.008 & -0.036 & -0.033 \\
\hline \multirow[t]{2}{*}{ X5 } & rp & & & & & 1.000 & $0.270 *$ & $0.966 * *$ & $-0.509 *$ & 0.161 & -0.135 & -0.119 & -0.044 & 0.111 & 0.041 & 0.024 & 0.026 \\
\hline & $\mathrm{rg}$ & & & & & 1.000 & 0.279 & 0.990 & -0.539 & 0.175 & -0.144 & -0.145 & -0.037 & 0.121 & 0.042 & 0.026 & 0.929 \\
\hline \multirow[t]{2}{*}{ X6 } & $\mathrm{rp}$ & & & & & & 1.000 & $0.263^{*}$ & $-0.360 * *$ & $0.261 * *$ & -0.024 & 0.115 & -0.044 & $0.291 *$ & $-0.255^{*}$ & $0.230 *$ & 0.232 \\
\hline & $\mathrm{rg}$ & & & & & & 1.000 & 0.275 & -0.381 & 0.286 & -0.007 & 0.129 & -0.054 & 0.301 & -0.280 & 0.248 & 0.256 \\
\hline \multirow[t]{2}{*}{ X7 } & $\mathrm{rp}$ & & & & & & & 1.000 & $-0.519 * *$ & 0.136 & -0.146 & -0.144 & -0.047 & 0.170 & 0.013 & -0.005 & -0.007 \\
\hline & $\mathrm{rg}$ & & & & & & & 1.000 & -0.547 & 0.145 & -0.166 & -0.165 & -0.040 & 0.172 & 0.014 & -0.005 & -0.0029 \\
\hline \multirow[t]{2}{*}{ X8 } & $\mathrm{rp}$ & & & & & & & & 1.000 & -0.295 & -0.090 & 0.003 & 0.146 & -0.070 & 0.011 & -0.156 & -0.142 \\
\hline & $\mathrm{rg}$ & & & & & & & & 1.000 & -0.301 & -0.106 & -0.007 & 0.160 & -0.064 & 0.010 & -0.168 & -0.152 \\
\hline \multirow[t]{2}{*}{ X9 } & $\mathrm{rp}$ & & & & & & & & & 1.000 & $0.333 * *$ & 0.150 & -0.140 & 0.120 & -0.004 & $0.765^{*}$ & $0.767 * *$ \\
\hline & $\mathrm{rg}$ & & & & & & & & & 1.000 & 0.356 & 0.160 & -0.170 & 0.126 & -0.004 & 0.783 & 0.469 \\
\hline \multirow[t]{2}{*}{ X10 } & $\mathrm{rp}$ & & & & & & & & & & 1.000 & $0.241 *$ & $-0.235^{*}$ & $-0.235^{*}$ & $-0.296^{*}$ & $0.415^{*}$ & $0.419 * *$ \\
\hline & $\mathrm{rg}$ & & & & & & & & & & 1.000 & 0.275 & -0.284 & -0.259 & -0.004 & 0.783 & 0.469 \\
\hline \multirow[t]{2}{*}{ X11 } & $\mathrm{rp}$ & & & & & & & & & & & 1.000 & $0.538 * *$ & -0.120 & 0.109 & $0.697 *$ & $0.695 * *$ \\
\hline & $\mathrm{rg}$ & & & & & & & & & & & 1.000 & 0.606 & -0.137 & 0.118 & 0.929 & 0.734 \\
\hline \multirow[t]{2}{*}{ X12 } & $\mathrm{rp}$ & & & & & & & & & & & & 1.000 & -0.062 & $0.205^{*}$ & $0.257 *$ & $0.258 * *$ \\
\hline & $\mathrm{rg}$ & & & & & & & & & & & & 1.000 & -0.072 & 0.226 & 0.270 & 0.279 \\
\hline \multirow[t]{2}{*}{ X13 } & $\mathrm{rp}$ & & & & & & & & & & & & & 1.000 & 0.109 & -0.019 & -0.016 \\
\hline & $\mathrm{rg}$ & & & & & & & & & & & & & 1.000 & 0.099 & -0.020 & -0.012 \\
\hline \multirow[t]{2}{*}{ X14 } & $\mathrm{rp}$ & & & & & & & & & & & & & & 1.000 & 0.015 & 0.005 \\
\hline & $\mathrm{rg}$ & & & & & & & & & & & & & & 1.000 & 0.020 & 0.003 \\
\hline \multirow[t]{2}{*}{ X15 } & $\mathrm{rp}$ & & & & & & & & & & & & & & & 1.000 & 0.996 \\
\hline & $\mathrm{rg}$ & & & & & & & & & & & & & & & 1.000 & 0.026 \\
\hline \multirow[t]{2}{*}{ X16 } & $\mathrm{rp}$ & & & & & & & & & & & & & & & & 1.000 \\
\hline & $\mathrm{rg}$ & & & & & & & & & & & & & & & & 1.000 \\
\hline
\end{tabular}

*Significant at $5 \%$ level of significance, $\quad$ **Significant at $1 \%$ level of significance $\mathrm{rp}=$ phenotypic correlation, $\mathrm{rg}=\mathrm{genotypic}$ correlation

X1=Plant height 30DAS, X2=Plant height 60DAS, X3=Plant height 90DAS, X4=Days to first flowering, X5=Days to fifty percent flowering, X6=First fruiting node, X7=Days to first fruiting, $X 8=$ =Internodal length, $X 9=$ Number of fruits per plant, $X 10=$ Fruit length, $X 11=$ Average fruit weight, $X 12=$ Fruit diameter, $X 13=$ Number of branches per plant, $\mathrm{X} 14=$ Number of seeds per fruit, $\mathrm{X} 15=$ Yield per hectare, $\mathrm{X} 16=$ Yield per plant 
Table.3 Genotypic and phenotypic path coefficient analysis for yield and its components in okra

\begin{tabular}{|c|c|c|c|c|c|c|c|c|c|c|c|c|c|c|c|c|}
\hline & & X1 & $\mathrm{X} 2$ & X3 & $\mathrm{X} 4$ & X5 & X6 & X7 & X8 & X9 & X10 & X11 & X12 & X13 & X14 & X15 \\
\hline \multirow[t]{2}{*}{$\mathrm{X} 1$} & $\mathrm{P}$ & -0.086 & -0.040 & -0.039 & 0.012 & 0.016 & -0.007 & 0.013 & 0.003 & -0.028 & -0.020 & -0.015 & 0.007 & 0.002 & 0.001 & -0.030 \\
\hline & $\mathrm{G}$ & 0.781 & 0.655 & 0.563 & -0.144 & -0.166 & 0.100 & -0.141 & -0.053 & 0.308 & 0.224 & 0.175 & -0.056 & -0.021 & -0.137 & 0.332 \\
\hline \multirow[t]{2}{*}{$\mathrm{X} 2$} & $\mathrm{P}$ & 0.008 & 0.018 & 0.012 & 0.001 & 0.001 & 0.006 & 0.001 & -0.003 & 0.004 & 0.001 & 0.005 & 0.002 & 0.004 & -0.001 & 0.006 \\
\hline & $\mathrm{G}$ & -0.639 & -0.761 & -0.539 & -0.035 & 0.020 & -0.315 & -0.009 & 0.151 & -0.233 & 0.059 & -0.240 & -0.129 & -0.237 & 0.038 & -0.297 \\
\hline \multirow[t]{2}{*}{ X3 } & $\mathrm{P}$ & -0.013 & -0.018 & -0.029 & -0.006 & -0.007 & -0.015 & -0.007 & 0.010 & -0.001 & -0.004 & -0.007 & 0.001 & -0.002 & 0.001 & -0.011 \\
\hline & $\mathrm{G}$ & -0.514 & -0.505 & -0.713 & -0.189 & -0.164 & -0.429 & -0.189 & 0.288 & -0.322 & -0.131 & -0.173 & 0.018 & -0.084 & 0.034 & -0.319 \\
\hline \multirow[t]{2}{*}{$\mathrm{X} 4$} & $\mathrm{P}$ & -0006 & 0.001 & 0.009 & 0.045 & 0.042 & 0.012 & 0.044 & -0.023 & 0.005 & -0.008 & -0.008 & -0.003 & 0.008 & 0.001 & -0.002 \\
\hline & $\mathrm{G}$ & -0.663 & 0.166 & 0.953 & 3.591 & 3.523 & 0.987 & 3.603 & -1.965 & 0.429 & -0.750 & -0.665 & -0.258 & 0.651 & -0.028 & -0.132 \\
\hline \multirow[t]{2}{*}{ X5 } & $\mathrm{P}$ & -0.051 & 0.004 & 0.061 & 0.249 & 0.266 & 0.072 & 0.257 & -0.136 & 0.043 & -0.036 & -0.032 & -0.012 & 0.030 & 0.011 & 0.007 \\
\hline & G & 0.253 & 0.032 & -0.273 & -1.162 & -1.184 & -0.331 & -1.173 & 0.639 & -0.208 & 0.171 & 0.172 & 0.044 & -0.143 & -0.050 & -0.031 \\
\hline \multirow[t]{2}{*}{ X6 } & $\mathrm{P}$ & -0.001 & -0.003 & -0.005 & -0.003 & -0.003 & -0.010 & -0.003 & 0.003 & -0.003 & 0.001 & -0.001 & 0.001 & -0.003 & 0.002 & -0.002 \\
\hline & $\mathrm{G}$ & 0.171 & 0.552 & 0.802 & 0.366 & 0.372 & 1.333 & 0.367 & -0.508 & 0.382 & -0.096 & 0.173 & -0.073 & 0.402 & -0.373 & 0.331 \\
\hline \multirow[t]{2}{*}{ X7 } & $\mathrm{P}$ & 0.028 & -0.003 & -0.045 & -0.183 & -0.182 & -0.050 & -0.188 & 0.098 & -0.026 & 0.028 & 0.027 & 0.009 & -0.032 & -0.002 & 0.002 \\
\hline & $\mathrm{G}$ & 0.567 & -0.037 & -0.830 & -3.141 & -3.102 & -0.863 & -3.130 & 1.714 & -0.456 & 0.521 & 0.518 & 0.125 & -0.540 & -0.044 & 0.017 \\
\hline \multirow[t]{2}{*}{ X8 } & $\mathrm{P}$ & -0.008 & -0.037 & -0.082 & -0.120 & -0.122 & -0.086 & -0.124 & 0.239 & -0.072 & -0.022 & 0.001 & 0.035 & -0.017 & 0.003 & -0.003 \\
\hline & $\mathrm{G}$ & 0.078 & 0.227 & 0.460 & 0.624 & 0.615 & 0.434 & 0.624 & -1.140 & 0.343 & 0.121 & 0.008 & -0.182 & 0.073 & -0.012 & 0.192 \\
\hline \multirow[t]{2}{*}{ X9 } & $\mathrm{P}$ & 0.158 & 0.114 & 0.188 & 0.049 & 0.079 & 0.127 & 0.066 & -0.145 & 0.485 & 0.162 & 0.073 & -0.068 & 0.039 & -0.002 & 0.373 \\
\hline & G & -5.525 & -4.292 & -6.314 & -1.671 & -2.457 & -4.007 & -2.040 & 4.217 & -13.98 & -4.979 & -2.243 & 2.423 & -1.767 & 0.062 & -10.96 \\
\hline \multirow[t]{2}{*}{$\mathbf{X 1 0}$} & $\mathrm{P}$ & 0.020 & 0.001 & 0.014 & -0.015 & -0.012 & -0.002 & -0.013 & -0.008 & 0.029 & 0.088 & 0.021 & -0.0021 & -0.021 & -0.026 & 0.037 \\
\hline & G & -0.541 & 0.148 & -0.346 & 0.394 & 0.273 & 0.012 & 0.314 & 0.201 & -0.671 & -1.887 & -0.519 & 0.536 & 0.48 & 0.604 & -0.891 \\
\hline \multirow[t]{2}{*}{ X11 } & $\mathrm{P}$ & 0.065 & 0.101 & 0.088 & -0.064 & -0.045 & 0.043 & -0.054 & 0.001 & 0.057 & 0.090 & 0.375 & 0.235 & -0.068 & 0.041 & 0.262 \\
\hline & G & -2.682 & -3.771 & -2.904 & 2.209 & 1.734 & -1.546 & 1.973 & 0.087 & -1.911 & -3.280 & -11.91 & -7.226 & 2.231 & -1.412 & -8.696 \\
\hline \multirow[t]{2}{*}{$\mathrm{X} 12$} & $\mathrm{P}$ & -0.006 & 0.008 & -0.001 & -0.005 & -0.004 & -0.004 & -0.004 & 0.012 & -0.011 & -0.019 & 0.043 & 0.081 & -0.005 & 0.017 & 0.021 \\
\hline & G & 0.074 & -0.195 & 0.030 & 0.082 & 0.043 & 0.063 & 0.046 & -0.184 & 0.199 & 0.3266 & -0.697 & -1.149 & 0.083 & -0.259 & -0.310 \\
\hline \multirow[t]{2}{*}{ X13 } & $\mathrm{P}$ & -0.002 & 0.019 & 0.007 & 0.016 & 0.010 & 0.027 & 0.016 & -0.006 & 0.011 & -0.021 & -0.016 & -0.006 & 0.091 & 0.010 & -0.002 \\
\hline & G & 0.022 & -0.254 & -0.096 & -0.147 & -0.098 & -0.245 & -0.140 & 0.052 & -0.103 & 0.211 & 0.152 & 0.058 & -0.814 & -0.080 & 0.017 \\
\hline \multirow[t]{2}{*}{ X14 } & $\mathrm{P}$ & 0.029 & 0.010 & 0.010 & 0.002 & -0.009 & 0.057 & -0.003 & -0.003 & 0.001 & 0.067 & -0.025 & -0.046 & -0.025 & -0.025 & -0.004 \\
\hline & $\mathrm{G}$ & -0.213 & -0.061 & -0.058 & -0.009 & 0.051 & -0.341 & 0.017 & 0.012 & -0.005 & -0.389 & 0.144 & 0.275 & 0.120 & 1.216 & 0.025 \\
\hline \multirow[t]{2}{*}{ X15 } & $\mathrm{P}$ & -0.030 & 0.006 & -0.011 & -0.002 & 0.007 & -0.002 & 0.002 & -0.003 & 0.373 & 0.037 & 0.262 & 0.021 & -0.002 & -0.004 & 0.935 \\
\hline & $\mathrm{G}$ & 0.332 & -0.297 & -0.319 & -0.132 & -0.031 & 0.331 & 0.017 & 0.192 & -10.96 & -0.891 & -8.696 & -0.310 & 0.017 & 0.025 & 1.320 \\
\hline
\end{tabular}

Residual effect at genotypic level: 0.1846, Residual effect at phenotypic level: 0.0745

$\mathrm{P}=$ Phenotypic, $\mathrm{G}=$ Genotypic

$\mathrm{X} 1=$ Plant height 30DAS, X2=Plant height 60DAS, X3=Plant height 90DAS, X4=Days to first flowering, X5=Days to fifty percent flowering, X6=First fruiting node, X7=Days to first fruiting, $X 8=$ Internodal length, $X 9=$ Number of fruits per plant, $X 10=$ Fruit length, $X 11=$ Average fruit weight, $X 12=$ Fruit diameter, $X 13=$ Number of branches per plant, $\mathrm{X} 14=$ Number of seeds per fruit, X15=Yield per hectare 
Most of the qualitative traits of $360-\mathrm{R}$ were comparable with the commercial check Arka Anamika in terms of characters namely dark green fruit colour, medim pubescence and acute fruit shape. The entry $360-\mathrm{R}$ had higher yield by $37.46 \%$ as compared to Arka Abhay. The performance of the entry $360-\mathrm{R}$ should be studied across the seasons for its stability. If found stable this could be released as an alternative to Arka Anamika. This kind of results were expressed by Prakash et al., (2001), Senapati et al., (2011), Thirupathi et al., (2012), Kumar et al., (2019) and Nesru et al., (2020) in okra. The polygenic trait such as yield is a result of characters namely number of fruits per plant and fruit weight. In the present study significantly higher number of fruits was recorded in the entry 360-R (22.50). This indicates that number of fruits trait was important for getting higher yield and there was only one entry namely $29-2-2$ which was at par with this. The entry $360-\mathrm{R}$ had $17.18 \%$ more number of fruits per plant as compared to Arka Abhay and $16.58 \%$ as compared to the check variety Arka Anamika. Similar findings were reported by Ajimal et al., (1979), Dhall et al., (2000), Sureshbabu et al., (2004), Sanjay et al., (2012) and Mishra et al., (2016). Significantly higher average fruit weight $(21.92 \mathrm{~g})$ was recorded in the entry $360-\mathrm{R}$ and there were two genotypes namely IC-001543 and Arka Anamika which were at par with this. The entry 360-R had $30.55 \%$ more fruit weight as compared to check variety Arka Abhay. So higher is the fruit weight higher is the yield. Hence it is important trait for increasing the yield. Similar results were expressed by Saryam et al., (2017) and Kumar et al., (2019).

In the present investigation both genotypic and phenotypic correlations were worked out for yield and its contributing characters. In general, genotypic correlation was higher than phenotypic correlations for eight characters studied. Number of fruits per plant $\left(r_{p}=\right.$ $\left.0.767^{* *}, r_{\mathrm{g}}=0.469\right)$, had highly significant phenotypic and genotypic association with fruit yield per plant followed by average fruit weight $\left(r_{p}=0.696^{* *}, r_{g}=0.734\right)$, fruit length $\left(r_{p}=0.419^{* *}, r_{g}=0.469\right)$, plant height 90DAS $\left(r_{p}=0.356^{* *}, r_{g}=0.442\right)$, plant height 30 DAS $\left(r_{p}=0.339^{* *}, r_{\mathrm{g}}=0.419\right)$, plant height 60DAS $\left(r_{p}=0.309^{* *}, r_{g}=0.386\right)$ and fruit diameter $\left(r_{p}=0.258 * *, r_{g}=0.279\right)$. The genotypes such as 360-R, 29-2-2, IC-014096, IC-001543, IC-0113356, IC-00795, IC-04328, IC-42490, B-3-39-R, IC-29119 and B-3-14922 were superior entries for the characters Yield per plant, number of fruits per plant, average fruit weight, fruit length and plant height had positive and significant correlation with fruit yield per plant. This indicates the possibility of increasing the yield by selecting for these characters. Thus, direct selection for above parameters will be useful in enhancing fruit yield of okra. These correlation outcomes are in line with works of Singh et al., (1974), Ajimal et al., (1979), Singh and Singh (1979), Bendale et al., (2003), Verma et al., (2007), Kumar et al., (2009), Mishra et al., (2016), Saryam et al., (2017), Kumar et al., (2019) and Nesru et al., (2020) in okra.

In present study path coefficient analysis exhibited that, the number of fruits per plant had highest positive direct effect on fruit yield per plant followed by average fruit weight, days to fifty per cent flowering, internodal length, number of branches per plant, fruit length, fruit diameter, days to first flowering and plant height 60 DAS, which further indicates the positive correlation between these characters. The characters which are showing higher positive effect could be used to select genotypes (360-R, IC-014096, 29-22, IC-001543, IC-42490, IC-111515, IC009856, RHBG-43, RHBG-175-41, IC755647, IC-011533, IC-29119, IC-013995 and B-3-39-R) for enhancement of fruit yield in okra. Similar results were reported by Ajimal et al., (1979), Reddy et al., (1985), 
Dhall et al., (2000), Sureshbabu et al., (2004), Mehta et al., (2006), Sateesh et al., (2011), Saryam et al., (2017), Sundaram (2018), Arun et al., (2019) and Nesru et al., (2020) in okra.

Any deviations in the results observed between the present study and other authors is attributed to the differences in the genotype used in the study, differences in number of genotypes, breeding methods employed, methods of statistical analysis adapted, soil characters and environmental conditions.

\section{References}

Ajimal, H. R., Rattan, R. S. and Saini, S. S., 1979. Correlation and path coefficient analysis in okra [Abelmoschus esculentus (L.) Moench]. Haryana Journal of Horticultural Sciences. 8 (1-2): 58-63.

Arun, K., Mukesh, K., Rakesh, V. and Sharma, M., 2019. Genetic variability, heritability and genetic advance studies of okra [(Abelmoschus esculentus (L.) Monech]. Journal of Pharmacognosy and Phytochemistry. 8(1): 1285-1290.

Bendale, V. W., Kadam, S. R., Bhave, S. G., Mehta J. L. and Pethe, U. B., 2003. Genetic variability and correlation studies in okra. Orissa Journal of Horticulture. 31 (2): 1-4.

Chauhan, D. V. S., 1972. Vegetable Production in India (third Edition), Ramprasad and sons. Agra. 6: 164172.

Dhall, R. K., Arora, S. K. and Rani, M., 2000, Correlation and path analysis in advanced generations of okra [Abelmoschus esculentus (L.) Moench.]. Indian Journal of Horticulture. 57(4): 342-346.

Johnson, H. W., Robinson, H. F. and Comstock, R. E., 1955. Estimates of genetic and environmental variability of soybeans. Agronomy Journal. 47(1):
314-318.

Kumar, S., Annapurna and Yadav, Y. C., 2009. Correlation coefficient and path analysis studies in okra [Abelmoschus esculentus (L.) Moench]. Annals of Horticulture. 2(2): 166-170.

Kumar, A., Kumar, M., Sharma, V. R., Singh, M. K., Singh, B. and Chand, P., 2019. Genetic Variability, Heritability and Genetic Advance studies in Genotypes of Okra [(Abelmoschus esculentus (L.) Moench]. Journal of Pharmacognosy and Phytochemistry. 8(1): 1285-1290.

Mehta, D. R., Dhaduk, L. K. and Patel, K. D., 2006. Genetic variability, correlation and path analysis studies in okra (Abelmoschus esculentus (L.) Moench). Agricultural Science Digest. 26 (1): $15-18$.

Mishra, K., Archana, M. and Senapati, N., 2016. Genetic variability and correlation studies in okra. Electronic Journal of Plant Breeding. 6(3): 866869.

Nesru, T., Wassu, M. and Shimelis, A., 2020. Agro morphological characterization and evaluation of okra [Abelmoschus esculentus (L.) Moench] genotypes for yield and other variability components at Melkassa, Central Ethiopia. MOJ Ecology and Environmental Sciences. 5 (2): 80-87.

Niranjan, R. S. and Mishra, M. N., 2003. Correlation and path coefficient analysis in okra Abelmoschus esculentus (L.) Moench. Progressive Horticulture. 35 (2): 192 -195.

Prakash, M., Kannan, K., Kumar, J. S., Bharthiveermani, B., Balaji, P. and Ganesan, J., 2001. Studies on the genetics of certain quantitative characters with particular reference to seed production in okra [Abelmoschus esculentus (L.) Moench]. Annals of Agricultural Research. 22 (1): 80 - 82.

Reddy, K. R., Singh, R. P., and Rai, A. K., 
1985. Variability and association analysis in okra. Madras Agricultural Journal. 72 (8): 478 -480.

Sanjay, K., Annapurna, Yogesh, C. Y. and Raghvendra, 2012. Genetic variability, heritability, genetic advance, correlation and path analysis in okra. Horticulture Flora Research Spectrum. 1(2): 139-144.

Saryam, D. K., Mittra, S. K., Mehta, A, K. and Sunil, P., 2017. Variation in genetic diversity in okra (Abelmoschus esculentus L.). Journal of functional and Environmental Botany. 7 (1): 5764.

Satish, A., Shanthakumar, G., Gangashetty, P. I., and Salimath, P. M., 2011. Association studies in okra (Abelmoschus esculentus (L.) Moench). Electronic Journal of Plant Breeding. 2(4): 568-573.

Senapati, N., Mishra, H. N, Beura, S. K., Dash, S. K., Prasad, G. and Patnaik, A., 2011. Genetic analysis in okra hybrids. Environment and Ecology. 29(3A): 1240 -1244.

Singh, K., Malik, Y., Kalloo, S. and Mahrotra, N., 1974. Genetic variability and correlation studies in bhindi [Abelmoschus esculentus (L). Moench]. Vegetable Science. 19 (1): $45-47$.

Singh, S. P. and Singh, H. N., 1979. Path coefficient analysis for yield components in okra. Indian Journal of Agricultural Sciences. 49 (4): 244-246.

Sundaram, 2018. Genetic variability, heritability and genetic advance studies in okra (Abelmoschus esculentus L.). International Journal of Chemical Studies. 6 (3): 3319-3321.

Sureshbabu, K. V., Gopalakrishnan, T. R. and Saly K. M., 2004. Genetic variability, correlation studies, path analysis and reaction to yellow vein mosaic virus (YVMV) in Abelmoschus caillei. Abstracts of first Indian Horticulture Congress, New Delhi, pp. 85- 86.

Thirupathi, R. M., Hari, B. K., Ganesh, M, Chandrasekhar, R. K., Begum, H., Purushothama, R, B. and Narshimulu, G., 2012. Genetic variability analysis for the selection of elite genotypes based on pod yield and quality from the germplasm of okra (Abelmoschus esculentus L.). Journal of Agricultural Technology. 8(2): 639-655.

Verma, B. K., Naidu, A. K. and Bajpai, H. K., 2007. Correlation and path coefficient analysis in okra [Abelmoschus esculentus (L.) Moench]. Abstract of International Conference on sustainable Agriculture for food, Bioenergy and livelihood security. Jabalpur. Feb 14-16, Vol. II: 463.

Wright, S., 1921. Correlation and causation. Journal of Agriculture Research. 20: 557-580.

Zeven, A. C. and Zhukovsky, P. M. 1975, Dictionary of cultivated plants and their centres of diversity. Centre for Agricultural Publishing and Documentation, Wageningen. p. 68.

\section{How to cite this article:}

Kiran Kumar, R. and Ramangouda V. Patil. 2020. Mean Performance, Character Association and Path Analysis Studies for Quantitative Characters in Okra (Abelmoschus esculentus (L.) Moench) Genotypes. Int.J.Curr.Microbiol.App.Sci. 9(11): 1357-1365. doi: https://doi.org/10.20546/ijcmas.2020.911.160 